\title{
PROJECTION MULTILEVEL METHODS FOR QUASILINEAR ELLIPTIC PARTIAL DIFFERENTIAL EQUATIONS: THEORETICAL RESULTS
}

\author{
THOMAS A. MANTEUFFEL ${ }^{\ddagger}$, STEPHEN F. MCCORMICK ${ }^{\ddagger}$, AND OLIVER RÖHRLE ${ }^{\dagger}$
}

\begin{abstract}
In a companion paper [8], we propose a new multilevel solver for two-dimensional elliptic systems of partial differential equations (PDEs) with nonlinearity of type $u \partial v$. The approach is based on a multilevel projection method (PML [9]) applied to a first-order system least-squares (FOSLS) functional that allows us to treat the nonlinearity directly. While [8] focuses on computation, here we concentrate on developing a theoretical framework that confirms optimal two-level convergence. To do so, we choose a first-order formulation of the Navier-Stokes equations as a basis of our theory. We establish continuity and coercivity bounds for the linearized Navier-Stokes equations and the full nonquadratic least-squares functional, as well as existence and uniqueness of a functional minimizer. This leads to the immediate result that one cycle of the two-level PML method reduces the functional norm by a factor that is uniformly less than 1 .
\end{abstract}

Key words. Projection Method, Multigrid, Least Squares, Finite Elements, Quasilinear PDEs, Navier-Stokes

AMS subject classifications. 35J60, 65N12, 65N30, 65N55

1. Introduction. Our companion paper [8] introduces a new multilevel solver for two-dimensional elliptic systems of partial differential equations (PDEs) with nonlinearity of type $u \partial v$. The approach is based on a multilevel projection method (PML [9]) applied to a first-order system least-squares (FOSLS) functional, where the nonlinearity is treated directly, with no need for linearization anywhere in the algorithm. While [8] focuses on computation, the key objective of the present paper is to establish local well-posedness of our functional minimization problem. This result leads to the immediate conclusion that our two-level solver converges linearly with grid independent factors, as observed numerically in [8]. This two-grid result can be extended to $W$-cycles in the usual way. However, an important alternative would be to establish a V-cycle result based on the general theory developed in [11] and [12]. This alternative would naturally yield grid-dependent convergence bounds because of the weak smoothness assumptions on the problem formulation (i.e., only Lipschitz continuity on the domain boundary).

We base our theory for a two-level PML method on the first-order formulation of the Navier-Stokes formulation given in (2.1) below. Although we choose this formulation as a foundation for our theoretical framework, it is not limited to it: similar results can be established for other PDEs of this class.

This paper is organized in the following way. Section 2 provides the first-order system formulation, with definitions, notation, and description of one two-level PML

\footnotetext{
${ }^{\dagger}$ Bioengineering Institute, The University of Auckland, Private Bag 92019, Auckland 1, New Zealand. Email: o.rohrle@acukland.ac.nz. This work was sponsored by the Department of Energy under grant numbers DE-FC02-01ER25479 and DE-FG02-03ER25574, Lawrence Livermore National Laboratory under contract number B533502, Sandia National Laboratory under contract number 15268, and the National Science Foundation under VIGRE grant number DMS-9810751.

‡Department of Applied Mathematics, Campus Box 526, University of Colorado at Boulder, Boulder, CO, 80309-0526. Email: \{tmanteuf, stevem\}@colorado.edu. This work was sponsored by the Department of Energy under grant numbers DE-FC02-01ER25479 and DE-FG02-03ER25574, Lawrence Livermore National Laboratory under contract number B533502, Sandia National Laboratory under contract number 15268, and the National Science Foundation under VIGRE grant number DMS-9810751.
} 
cycle step. Section 3 establishes several continuity and coercivity bounds for the Oseen equations, as well as for the full nonquadratic least-squares functional. Section 4 shows existence and uniqueness of a functional minimizer, some characteristics of coarse-grid correction and relaxation, and two-grid convergence.

2. First-Order System Formulation, Definitions, Notation, and Other Preliminaries. We use $c$ and $C$ throughout as generic constants that my change value with every occurrence but are independent of mesh size. To keep track of a specific value for a constant, subindices may be used.

First-order system least-squares formulations for the Navier-Stokes equations are discussed in $[1,2,3,6]$. In the framework of this paper, we consider the first-order velocity-flux formulation of the Navier-Stokes equations given in [1] and [2]:

$$
\mathcal{L}(\mathbf{x})=\mathrm{g}:=\left\{\begin{aligned}
\nabla \mathbf{u}^{t}-\mathbf{U}=\mathbf{0} & \text { in } \Omega, \\
-(\nabla \cdot \mathbf{U})^{t}+\nabla p+R e \mathbf{U}^{t} \mathbf{u}=\mathbf{f} & \text { in } \Omega, \\
\nabla \cdot \mathbf{u}=0 & \text { in } \Omega, \\
\nabla \times \mathbf{U}=\mathbf{0} & \text { in } \Omega, \\
\nabla(t r \mathbf{U})=\mathbf{0} & \text { in } \Omega,
\end{aligned}\right.
$$

where $\Omega$ is a subset of $\mathbb{R}^{n}(n=2,3)$ with Lipschitz continuous boundary $\partial \Omega$ and $\mathbf{f} \in L^{2}(\Omega)^{n}$. As boundary conditions, without loss of generality, we take $\mathbf{u}=\mathbf{0}$ and $\mathbf{n} \times \mathbf{U}=\mathbf{0}$ on $\partial \Omega$, where $\mathbf{n}$ is the outward unit normal on $\partial \Omega$. Writing the unknowns as $\mathbf{x}=(\mathbf{u}, \mathbf{U}, p)$, then the nonquadratic functional is constructed by taking the $L^{2}$-norm of each interior equation:

$$
\mathcal{F}(\mathbf{x} ; \mathrm{g})=\|\mathcal{L}(\mathbf{x})-\mathrm{g}\|_{0, \Omega}^{2}, \quad \mathbf{x} \in \mathcal{V},
$$

where $\mathrm{g}=(\mathbf{0}, \mathbf{f}, 0, \mathbf{0}, \mathbf{0})^{T}$ and the space is defined by

$$
\mathcal{V}=H_{0}^{1}(\Omega)^{n} \times \mathcal{V}_{0} \times\left(H^{1}(\Omega) / \mathbb{R}\right),
$$

with

$$
\mathcal{V}_{0}=\left\{\mathbf{U} \in H^{1}(\Omega)^{n^{2}}: \mathbf{n} \times \mathbf{U}=\mathbf{0} \text { on } \partial \Omega\right\} .
$$

It is shown in [5] that the Navier-Stokes equations generally have more than one solution, unless the viscosity and the external forces satisfy very stringent requirements. However, it can also be shown that, in many practical examples, these solutions are mostly isolated, i.e., there exist a neighborhood in which each solution is unique. Bifurcation phenomena are rare. We thus assume we are in a closed neighborhood, $\overline{\mathcal{B}}\left(\mathbf{x}_{*}, r\right)$, of an isolated solution, $\mathbf{x}_{*} \in \mathcal{V}$, to $(2.1)$, that is, a global minimum of (2.2), for which $\mathcal{F}\left(\mathbf{x}_{*} ; \mathrm{g}\right)=0$. The neighborhood is taken to be an $H^{1}$-ball around $\mathbf{x}_{*}$ with radius $r>0$ defined as

$$
\mathcal{B}\left(\mathbf{x}_{*}, r\right):=\left\{\mathbf{x} \in \mathcal{V}:\left\|\mathbf{x}-\mathbf{x}_{*}\right\|_{1, \Omega}<r\right\},
$$

where

$$
\|\mathbf{x}\|_{1, \Omega}^{2} \equiv\|\mathbf{u}\|_{1, \Omega}^{2}+\|\mathbf{U}\|_{1, \Omega}^{2}+\|p\|_{1, \Omega}^{2} .
$$


Its closure is $\overline{\mathcal{B}}\left(\mathbf{x}_{*}, r\right)=\left\{\mathbf{x} \in \mathcal{V}:\left\|\mathbf{x}-\mathbf{x}_{*}\right\|_{1, \Omega} \leq r\right\}$. Several places along the way, we assume that $r$ is so small that certain expansions we develop give us the desired bounds.

Denote by $\mathcal{L}^{\prime}(\mathbf{x})[\mathbf{y}]$ the first Fréchet derivative of operator $\mathcal{L}$ at $\mathbf{x} \in \mathcal{V}$ in direction $\mathbf{y}=(\mathbf{v}, \mathbf{V}, q) \in \mathcal{V}$. Note that the nonlinear term, $\operatorname{Re} \mathbf{U}^{t} \mathbf{u}$, in (2.1) becomes $\operatorname{Re}\left(\mathbf{V}^{t} \mathbf{u}+\right.$ $\left.\mathbf{U}^{t} \mathbf{v}\right)$ in $\mathcal{L}^{\prime}(\mathbf{x})[\mathbf{y}]$ and that $\mathcal{L}^{\prime}(\mathbf{x})[\mathbf{y}]$ is linear in $\mathbf{y}$. Also, $\mathcal{L}^{\prime}(\mathbf{x})$ is the same operator as that for the Oseen equations (cf. [7]). $\mathcal{L}^{\prime \prime}(\mathbf{x})[\mathbf{y}, \mathbf{z}]$ denotes the second Fréchet derivative at $\mathbf{x}$ in directions $\mathbf{y}$ and $\mathbf{z}=(\mathbf{w}, \mathbf{W}, t) \in \mathcal{V}$. For the linear terms of (2.1), the second Fréchet derivative is the zero operator. For the nonlinear term, we obtain $\operatorname{Re}\left(\mathbf{V}^{t} \mathbf{w}+\mathbf{W}^{t} \mathbf{v}\right)$, so $\mathcal{L}^{\prime \prime}(\mathbf{x})$ is independent of $\mathbf{x}$.

Another set definition we use later is the closed line segment connecting points $\mathbf{x}, \mathbf{y} \in \mathcal{V}:[\mathbf{x}, \mathbf{y}]:=\{\theta \mathbf{x}+(1-\theta) \mathbf{y}: 0 \leq \theta \leq 1\}$. This notation should not be confused with the square brackets used for directional derivatives because the operator always immediately precedes the direction.

Having defined the first and second Fréchet derivatives for operator $\mathcal{L}$, we are able to express the first and second Fréchet derivatives of the nonquadratic functional in (2.2) in terms of $\mathcal{L}$ and its derivatives. For $\mathbf{x}, \mathbf{y} \in \mathcal{V}$, the first Fréchet derivative of $(2.2)$ in direction $\mathbf{y}$ is

$$
\mathcal{F}^{\prime}(\mathbf{x} ; \mathrm{g})[\mathbf{y}]=2<\mathcal{L}(\mathbf{x})-\mathrm{g}, \mathcal{L}^{\prime}(\mathbf{x})[\mathbf{y}]>
$$

Its second Fréchet derivative in direction $[\mathbf{y}, \mathbf{y}]$ (needed later for Taylor expansions) is

$$
\mathcal{F}^{\prime \prime}(\mathbf{x} ; \mathrm{g})[\mathbf{y}, \mathbf{y}]=2\left\|\mathcal{L}^{\prime}(\mathbf{x})[\mathbf{y}]\right\|_{0, \Omega}^{2}+2<\mathcal{L}(\mathbf{x})-\mathrm{g}, \mathcal{L}^{\prime \prime}(\mathbf{x})[\mathbf{y}, \mathbf{y}]>
$$

REMARK 1. As with all multigrid schemes, relaxation is the basis for our PML approach. One choice is steepest descent, which involves a gradient direction, $\mathbf{d}$, in $\mathcal{V}$ and a step size, s, determined as the smallest nonnegative critical point of $\mathcal{F}(\mathbf{x}-s \mathbf{d} ; \mathrm{g})$. To understand this step, it is useful to examine the polynomial

$$
\begin{aligned}
\mathcal{F}(\mathbf{x}-s \mathbf{d} ; \mathrm{g})=\mathcal{F}(\mathbf{x} ; \mathrm{g})-s \mathcal{F}^{\prime}(\mathbf{x} ; \mathbf{g})[\mathbf{d}] & +\frac{s^{2}}{2} \mathcal{F}^{\prime \prime}(\mathbf{x} ; \mathbf{g})[\mathbf{d}, \mathbf{d}] \\
& -\frac{s^{3}}{6} \mathcal{F}^{\prime \prime \prime}(\mathbf{x} ; \mathbf{g})[\mathbf{d}, \mathbf{d}, \mathbf{d}]+\frac{s^{4}}{24} \mathcal{F}^{(4)}(\mathbf{x} ; \mathbf{g})[\mathbf{d}, \mathbf{d}, \mathbf{d}, \mathbf{d}] .
\end{aligned}
$$

From (2.4), we see that $\mathcal{F}^{\prime \prime \prime}(\mathbf{x} ; \mathbf{g})[\mathbf{d}, \mathbf{d}, \mathbf{d}]=6<\mathcal{L}^{\prime}(\mathbf{x})[\mathbf{d}], \mathcal{L}^{\prime \prime}(\mathbf{x})[\mathbf{d}, \mathbf{d}]>$ and $\mathcal{F}^{(4)}(\mathbf{x} ; \mathbf{g})[\mathbf{d}, \mathbf{d}, \mathbf{d}, \mathbf{d}]=6<\mathcal{L}^{\prime \prime}(\mathbf{x})[\mathbf{d}, \mathbf{d}], \mathcal{L}^{\prime \prime}(\mathbf{x})[\mathbf{d}, \mathbf{d}]>$. Thus, when $\mathcal{F}^{\prime \prime}(\mathbf{x} ; \mathbf{g})>0$, an inspection of this polynomial for $s>0$ implies that there exists a smallest nonnegative critical point, $s$, of $\mathcal{F}(\mathbf{x}-s \mathbf{d} ; \mathrm{g})$ and that it must be a local minimum of $\mathcal{F}(\mathbf{x}, \mathbf{g})$ such that $\mathcal{F}(\mathbf{x}-s \mathbf{d} ; \mathrm{g}) \leq \mathcal{F}(\mathbf{x} ; \mathrm{g})$. (Note that either $\mathbf{d}=\mathbf{0}$ and $\mathcal{F}(\mathbf{x}-s \mathbf{d} ; \mathrm{g})=\mathcal{F}(\mathbf{x} ; \mathrm{g})$, so $s=0$, or $\mathcal{F}(\mathbf{x}-s \mathbf{d} ; \mathrm{g})$ initially decreases, but then tends to $+\infty$ as $s$ goes from 0 to $\infty$.)

Similar definitions can be made for subspaces of $\mathcal{V}$. Consider a quasi-uniform finite element partition of $\Omega$ with approximate mesh size $h$ and let $H^{h}(\Omega)$ be the corresponding finite element subspace of $H^{1}(\Omega)$ consisting of piecewise polynomials: a function in $H^{h}(\Omega)$ is continuous on $\Omega$ and polynomial within each element. Let $H_{0}^{h}(\Omega)$ denote the subspace of $H^{h}(\Omega)$ of functions that are zero on $\partial \Omega$. Then define

$$
\mathcal{S}^{h}=H_{0}^{h}(\Omega)^{n} \times \mathcal{V}_{0}^{h} \times\left(H^{h}(\Omega) / \mathbb{R}\right) \subset \mathcal{V}
$$


with

$$
\mathcal{V}_{0}^{h}=\left\{\mathbf{U}^{h} \in H^{h}(\Omega)^{n^{2}}: \mathbf{n} \times \mathbf{U}^{h}=\mathbf{0} \text { on } \partial \Omega\right\} .
$$

Suppose also that we have a corresponding coarser $2 h$ level so that the corresponding discrete space, $\mathcal{S}^{2 h}$, forms a subspace of $\mathcal{S}^{h}$. For this paper, we assume standard nested finite element spaces, $S^{2 h} \subset S^{h} \subset \mathcal{V}$, that satisfy the approximation property,

$$
\inf _{\mathbf{x}^{2 h} \in \mathcal{S}^{2 h}}\left\|\mathbf{x}^{h}-\mathbf{x}^{2 h}\right\|_{0, \Omega}^{2} \leq C_{1} h^{2}\left\|\mathbf{x}^{h}\right\|_{1, \Omega}^{2}
$$

and the inverse estimate,

$$
\left\|\mathbf{x}^{h}\right\|_{1, \Omega}^{2} \leq \frac{C_{2}}{h^{2}}\left\|\mathbf{x}^{h}\right\|_{0, \Omega}^{2},
$$

for all $\mathrm{x}^{h}$ in $\mathcal{S}^{h}$, where $C_{1}$ and $C_{2}$ are positive constants that do not depend on $h$ (see [4]). Further, define a discrete $H^{1}$-ball by $\mathcal{B}^{h}\left(\mathbf{x}_{*}, r\right)=\left\{\mathbf{x}^{h} \in \mathcal{S}^{h}:\left\|\mathbf{x}^{h}-\mathbf{x}_{*}\right\|_{1, \Omega}<r\right\}$ and its closure by $\overline{\mathcal{B}}^{h}\left(\mathbf{x}_{*}, r\right)=\left\{\mathbf{x}^{h} \in \mathcal{S}^{h}:\left\|\mathbf{x}^{h}-\mathbf{x}_{*}\right\|_{1 \Omega} \leq r\right\}$. As we said, we choose $r$ progressively smaller in several places in what follows. Nowhere does this requirement depend on $h$. However, we implicitly assume that, no matter how small $r$ becomes, $h$ is so small that $\mathcal{B}^{h}\left(\mathbf{x}_{*}, r\right) \neq \emptyset$.

Before being able to define the relaxation scheme and two-level PML method, we introduce the discrete functional and its gradient as well as the operator norm associated with the second Fréchet derivative of functional $\mathcal{F}(\mathbf{x} ; \mathbf{g})$.

Definition 2.1 (Discrete Functional And its $L^{2}$-Gradient). Let $\mathbf{x}^{h} \in$ $\overline{\mathcal{B}}^{h}\left(\mathbf{x}_{*}, r\right)$ and define $\mathcal{F}^{h}\left(\mathbf{x}^{h} ; \mathrm{g}\right)$ as the restriction of $\mathcal{F}\left(\mathbf{x}^{h} ; \mathrm{g}\right)$ to space $\mathcal{S}^{h}$. Now let $\mathbf{y}^{h} \in \mathcal{S}^{h}$. By the definition of the first Fréchet derivative, we have

$$
\mathcal{F}^{h^{\prime}}\left(\mathbf{x}^{h} ; \mathrm{g}\right)\left[\mathbf{y}^{h}\right]=<\mathcal{L}\left(\mathbf{x}^{h}\right)-\mathrm{g}, \mathcal{L}^{\prime}\left(\mathbf{x}^{h}\right)\left[\mathbf{y}^{h}\right]>,
$$

and, since $\mathcal{S}^{h}$ is finite dimensional, the Riesz Representation Theorem guarantees the existence of the discrete $L^{2}$-gradient, $\nabla^{h} \mathcal{F}\left(\mathbf{x}^{h} ; \mathrm{g}\right) \in \mathcal{S}^{h}$, which satisfies

$$
\mathcal{F}^{h^{\prime}}\left(\mathbf{x}^{h} ; \mathrm{g}\right)\left[\mathbf{y}^{h}\right]=<\mathcal{L}^{\prime *}\left(\mathbf{x}^{h}\right)\left(\mathcal{L}\left(\mathbf{x}^{h}\right)-\mathrm{g}\right), \mathbf{y}^{h}>=:<\nabla^{h} \mathcal{F}\left(\mathbf{x}^{h} ; \mathrm{g}\right), \mathbf{y}^{h}>.
$$

Note that $\nabla^{h} \mathcal{F}\left(\mathbf{x}^{h} ; \mathrm{g}\right) \in \mathcal{S}^{h}$ can be defined weakly by $<\nabla^{h} \mathcal{F}\left(\mathbf{x}^{h} ; \mathrm{g}\right), \mathbf{y}^{h}>=<\mathcal{L}\left(\mathbf{x}^{h}\right)-$ $\mathrm{g}, \mathcal{L}^{\prime}\left(\mathbf{x}^{h}\right)\left[\mathbf{y}^{h}\right]>$, for all $\mathbf{y}^{h} \in \mathcal{S}^{h}$. Note also that $\nabla^{h} \mathcal{F}^{h}\left(\mathbf{x}^{h} ; \mathrm{g}\right)=\nabla^{h} \mathcal{F}^{h}\left(\mathbf{x}^{h} ; \mathrm{g}^{h}\right)$, where $\mathrm{g}^{h}$ is the $L^{2}$-orthogonal projection of $\mathrm{g}$ onto space $\mathcal{L}^{\prime}\left(\mathrm{x}^{h}\right) \mathcal{S}^{h}$.

REMARK 2. Denote by $\mathbf{x}_{*}^{h}$ the element in $\mathcal{S}^{h}$ that minimizes $(2.2)$ over $\overline{\mathcal{B}}^{h}\left(\mathbf{x}_{*}, r\right)$. Such an element exists because this set is compact and $\mathcal{F}(\mathbf{x} ; \mathrm{g})$ is continuous, as we show in Theorem 2. Note that if $\mathbf{x}^{h} \in \mathcal{B}^{h}\left(\mathbf{x}_{*}, r\right)$ (i.e., the interior of the ball), then $\mathbf{x}_{*}^{h}$ is a grid $h$ critical point in the sense that

$$
<\nabla^{h} \mathcal{F}\left(\mathbf{x}_{*}^{h} ; \mathrm{g}\right), \mathbf{y}^{h}>=<\mathcal{L}\left(\mathbf{x}_{*}^{h}\right)-\mathrm{g}, \mathcal{L}^{\prime}\left(\mathbf{x}_{*}^{h}\right)\left[\mathbf{y}^{h}\right]>=0,
$$

for all $\mathbf{y}^{h} \in \mathcal{S}^{h}$, provided $\mathcal{F}^{\prime \prime}(\mathbf{x} ; \mathbf{g})$ is bounded on $\mathcal{B}\left(\mathbf{x}_{*}, r\right)$, as we show in Theorem 1 below. This follows from a standard argument based on Taylor series and outlined as follows: if

$$
\begin{aligned}
0 \leq \mathcal{F}\left(\mathbf{x}_{*}^{h}-s \nabla^{h} \mathcal{F}\left(\mathbf{x}_{*}^{h} ; \mathrm{g}\right)\right)-\mathcal{F}\left(\mathbf{x}_{*}^{h} ; \mathrm{g}\right)= \\
\quad-s\left\|\nabla^{h} \mathcal{F}\left(\mathbf{x}_{*}^{h} ; \mathrm{g}\right)\right\|_{0, \Omega}^{2}+s^{2} \mathcal{F}^{\prime \prime}\left(\tilde{\mathbf{x}}^{h} ; \mathrm{g}\right)\left[\nabla^{h} \mathcal{F}\left(\mathbf{x}_{*}^{h} ; \mathrm{g}\right), \nabla^{h} \mathcal{F}\left(\mathbf{x}_{*}^{h} ; \mathrm{g}\right)\right] ;
\end{aligned}
$$


and, for small enough but positive s, we could make the last expression negative (a contradiction) unless $\nabla^{h} \mathcal{F}\left(\mathbf{x}_{*}^{h} ; \mathbf{g}\right)=0$. This standard argument is referred to later in the proof of Lemma 4.3 to show that the coarse-grid correction step of PML (described next) is determined by a grid 2 h critical point. That $\mathbf{x}_{*}$ is a critical point of $\mathcal{F}(\mathbf{x} ; \mathbf{g})$ in $\mathcal{B}\left(\mathbf{x}_{*}, r\right)$ follows simply from $(2.3)$.

Definition 2.2 (Discrete Operator Norm of the Second Derivative). Let $\mathbf{x}^{h} \in \overline{\mathcal{B}}^{h}\left(\mathbf{x}_{*}, r\right)$ and $\mathbf{y}^{h} \in \mathcal{S}^{h}$. Then the discrete operator norm associated with the second Fréchet derivative of functional $\mathcal{F}\left(\mathbf{x}^{h} ; \mathrm{g}\right)$ is defined by

$$
\left\|\left|\mathcal{F}^{\prime \prime}\left(\mathbf{x}^{h} ; \mathrm{g}\right) \|\right|_{0, h}=\sup _{0 \neq \mathbf{y}^{h} \in S^{h}} \frac{\left|\mathcal{F}^{\prime \prime}\left(\mathbf{x}^{h} ; \mathbf{g}\right)\left[\mathbf{y}^{h}, \mathbf{y}^{h}\right]\right|}{<\mathbf{y}^{h}, \mathbf{y}^{h}>}\right.
$$

Next, we define one step of relaxation. We consider two types of schemes, both of which use the discrete gradient as a descent direction. The first scheme reduces to Richardson for the linear case and the second is optimal steepest descent. The theory focuses on the Richardson-type scheme because it is simpler to analyze and it sets the stage for a simple conclusion for steepest descent.

Definition 2.3 (Relaxation). One step of Richardson-type relaxation is defined by

$$
\mathbf{x}^{h} \leftarrow \mathbf{x}^{h}-\frac{\omega}{\left.\left\|\mathcal{F}^{\prime \prime}\left(\mathbf{x}^{h} ; \mathrm{g}\right)\right\|\right|_{0, h}} \nabla^{h} \mathcal{F}\left(\mathbf{x}^{h} ; \mathrm{g}\right),
$$

where $\nabla^{h} \mathcal{F}\left(\mathbf{x}^{h} ; \mathrm{g}\right)$ is the search direction, $1 /\left\|\left|\mathcal{F}^{\prime \prime}\left(\mathbf{x}^{h} ; \mathrm{g}\right) \|\right|_{0, h}\right.$ the basic step length, and $\omega$ a damping parameter. One step of steepest descent is defined by

$$
\mathbf{x}^{h} \leftarrow \mathbf{x}^{h}-s \nabla^{h} \mathcal{F}\left(\mathbf{x}^{h} ; \mathrm{g}\right)
$$

where $s$ is chosen as the smallest nonnegative root of

$$
\frac{\partial \mathcal{F}}{\partial s}\left(\left(\mathbf{x}^{h}-s \nabla^{h} \mathcal{F}\left(\mathbf{x}^{h} ; \mathrm{g}\right)\right) ; \mathrm{g}\right)=0 \text {. }
$$

We now have all the ingredients needed to describe the two-level PML method. Its first step computes the nearest locally optimal coarse-grid correction, which Lemma 4.3 below shows must exist uniquely provided we are close enough to $\mathbf{x}_{*}$. Its second step is one relaxation sweep given by either (2.8) or (2.9). The method in (2.8) is well defined because $\mathcal{F}^{\prime \prime}\left(\mathrm{x}^{h} ; \mathrm{g}\right)$ is nonzero, as Theorem 1 below shows. The method in (2.9) is also well defined as Remark 1 shows.

STEP 1. For a given initial guess, $\mathbf{x}_{0}^{h} \in \mathcal{S}^{h}$, perform the coarse-grid correction step given by $\mathbf{x}_{\frac{1}{2}}^{h} \leftarrow \mathbf{x}_{0}^{h}+\mathbf{x}_{*}^{2 h}$, where $\mathbf{x}_{*}^{2 h}$ is the local minimizer of $\mathcal{F}\left(\mathbf{x}_{0}^{h}+\mathbf{x}^{2 h} ; \mathrm{g}\right)$ (e.g., it is a grid $2 \mathrm{~h}$ critical point) with minimal $H^{1}$-norm:

$$
\mathbf{x}_{*}^{2 h}=\underset{\mathbf{x}^{2 h} \in S^{2 h}}{\operatorname{argmin}}\left\{\left\|\mathbf{x}^{2 h}\right\|_{1, \Omega}: \nabla^{2 h} \mathcal{F}\left(\mathbf{x}_{0}^{h}+\mathbf{x}^{2 h} ; \mathrm{g}\right)=0, \mathcal{F}\left(\mathbf{x}_{0}^{h}+\mathbf{x}^{2 h} ; \mathrm{g}\right) \leq \mathcal{F}\left(\mathbf{x}_{0}^{h} ; \mathrm{g}\right)\right\} .
$$
to $\mathbf{x}_{\frac{1}{2}}^{h}$.

STEP 2. Let $\mathbf{x}_{1}^{h}$ be the result of one relaxation step given by (2.8) or (2.9) applied

Further iterations of PML are defined in the obvious way, with $\mathbf{x}_{k}^{h}$ taking on the role of $\mathbf{x}_{0}^{h}$ and $\mathbf{x}_{k+1}^{h}$ being the result corresponding to $\mathbf{x}_{1}^{h}$, for $k=1,2, \ldots$. 
3. Continuity and Coercivity bounds. In this section, we first establish continuity and coercivity for the Oseen equations (Lemma 3.3). We then use Lemmas 3.1 and 3.3 to prove continuity and coercivity of $\mathcal{F}^{\prime \prime}(\mathbf{x} ; \mathrm{g})[\mathbf{y}, \mathbf{y}]$ as a function of $\mathbf{y} \in \mathcal{V}$, for all $\mathbf{x} \in \overline{\mathcal{B}}\left(\mathbf{x}_{*}, r\right)$ (Theorem 1). The results in this section help us later to establish the key objective of our two-level method: one cycle of two-level PML reduces the functional norm by a factor that is bounded uniformly below 1 (Theorem 4).

Lemma 3.1. There exist a $\gamma_{0}$, depending only on Re and $\Omega$, such that

$$
\left\|\mathcal{L}^{\prime \prime}(\mathbf{x})[\mathbf{y}, \mathbf{z}]\right\|_{0, \Omega} \leq \gamma_{0}\|\mathbf{y}\|_{1, \Omega}\|\mathbf{z}\|_{1, \Omega}
$$

for all $\mathbf{x}, \mathbf{y}$, and $\mathbf{z}$ in $\mathcal{V}$.

Proof. Recall, for $\mathbf{x}=(\mathbf{u}, \mathbf{U}, p), \mathbf{y}=(\mathbf{v}, \mathbf{W}, q)$, and $\mathbf{z}=(\mathbf{w}, \mathbf{W}, t)$ in $\mathcal{V}$, that the second Fréchet derivative for the linear terms of (2.1) is the zero operator. For the nonlinear term, we obtain $\operatorname{Re}\left(\mathbf{V}^{t} \mathbf{w}+\mathbf{W}^{t} \mathbf{v}\right)$. Then, the result follows directly from the Sobolev Imbedding Theorem about multiplication in Sobolev spaces (Corollary I.1.1 in [5]).

Lemma 3.2. For all $\mathbf{x}=(\mathbf{u}, \mathbf{U}, p) \in \mathcal{V}$, there exist two positive constants, $\tilde{c}_{3}$ and $\tilde{C}_{3}$, depending only on $R e, \mathbf{x}$, and $\Omega$, such that

$$
\tilde{c}_{3}(\mathbf{x})\|\mathbf{y}\|_{1, \Omega}^{2} \leq\left\|\mathcal{L}^{\prime}(\mathbf{x})[\mathbf{y}]\right\|_{0, \Omega}^{2} \leq \tilde{C}_{3}(\mathbf{x})\|\mathbf{y}\|_{1, \Omega}^{2},
$$

for all $\mathbf{y}=(\mathbf{v}, \mathbf{V}, q) \in \mathcal{V}$.

Proof. We use the derivation of the regularity estimate, as well as Theorems $3.2,4.1$, and 4.2 in [7], as guidelines for the proof of this lemma. Analogous to the continuity and coercivity proof for $\mathcal{L}^{\prime}((\mathbf{u}, p))[(\mathbf{v}, \mathbf{V}, q)]$ in $[7]$, we start from the Oseen equations in the following form:

$$
\begin{aligned}
-\Delta \mathbf{v}+R e\left[\left(\nabla \mathbf{v}^{t}\right)^{t} \mathbf{u}+\mathbf{U}^{t} \mathbf{v}\right]+\nabla q & =\mathbf{f}, \\
\nabla \cdot \mathbf{v} & =g,
\end{aligned}
$$

where $g \in L^{2}(\Omega)$. The first equation differs from that in [7] because $\mathbf{U}$ is used instead of $\nabla \mathbf{u}^{t}$. We also relax the smoothness assumption by only requiring $\mathbf{u}$ and $\mathbf{U}$ to be in $H_{0}^{1}(\Omega)^{n}$ and $\mathcal{V}_{0}$, respectively. ([7] requires $\mathbf{u}$ to be in $\left.H_{0}^{2}(\Omega)^{n}\right)$.

First, we establish an a priori $H^{1}$-regularity estimate for the equations in (3.1): if $\Omega$ has Lipschitz boundary, then, for $\mathbf{f} \in H_{0}^{-1}(\Omega)^{n}$ and $g \in L_{0}^{2}(\Omega)$, the weak solution of $(3.1),(\mathbf{v}, q) \in H_{0}^{1}(\Omega)^{n} \times L_{0}^{2}(\Omega)$, satisfies the a priori estimate

$$
\left\|\nabla \mathbf{v}^{t}\right\|_{0, \Omega}+\|q\|_{0, \Omega} \leq \operatorname{const}\left(\|\mathbf{f}\|_{-1, \Omega}+\|g\|_{0, \Omega}+\|\mathbf{v}\|_{\delta_{\mathrm{o}}+\delta, \Omega}\right),
$$

for $\delta \in\left(0, \frac{1}{2}\right)$, where $\delta_{\mathrm{o}}=0$ for $\Omega \subset \mathbb{R}^{2}$ and $\delta_{\mathrm{o}}=\frac{1}{2}$ for $\Omega \subset \mathbb{R}^{3}$.

To prove this estimate, we first take the pointwise dot product of the first equation of (3.1) with any $\boldsymbol{\psi} \in H_{0}^{1}(\Omega)^{n}$ and the dot product of the second equation of (3.1) with any $\phi \in L^{2}(\Omega)$, integrate it over $\Omega$, and use integration by parts. This yields

$$
\begin{aligned}
<\nabla \mathbf{v}^{t}, \nabla \boldsymbol{\psi}^{t}>+R e<\left(\nabla \mathbf{v}^{t}\right)^{t} \mathbf{u}+\mathbf{U}^{t} \mathbf{v}, \boldsymbol{\psi}>-<q, \nabla \cdot \boldsymbol{\psi}>= & <\mathbf{f}, \boldsymbol{\psi}>, \\
<\nabla \cdot \mathbf{v}, \phi>= & <g, \phi>.
\end{aligned}
$$

Since $g \in L^{2}(\Omega)$, we can choose an $\mathbf{s} \in H_{0}^{1}(\Omega)^{n}$, according to Lemma 4.1 in [7], such that

$$
\nabla \cdot \mathbf{s}=g \quad \text { and } \quad|\mathbf{s}|_{1, \Omega} \leq C\|g\|_{0, \Omega} .
$$


Then, setting $\boldsymbol{v}=\mathbf{v}-\mathbf{s} \in H_{0}^{1}(\Omega)^{n}$ in (3.3), we have

$$
\left\{\begin{aligned}
&<\nabla \boldsymbol{v}^{t}, \nabla \boldsymbol{\psi}^{t}>+R e<\left(\nabla \boldsymbol{v}^{t}\right)^{t} \mathbf{u}+\mathbf{U}^{t} \boldsymbol{v}, \boldsymbol{\psi}>-<q, \nabla \cdot \boldsymbol{\psi}>=<\mathbf{f}, \boldsymbol{\psi}>-<\nabla \mathbf{s}^{t}, \nabla \boldsymbol{\psi}^{t}> \\
&-\operatorname{Re}<\left(\nabla \mathbf{s}^{t}\right)^{t} \mathbf{u}+\mathbf{U}^{t} \mathbf{s}, \boldsymbol{\psi}> \\
&<\nabla \cdot \boldsymbol{v}, \phi>=<0, \phi>
\end{aligned}\right.
$$

for any $\boldsymbol{\psi} \in H_{0}^{1}(\Omega)^{n}$ and $\phi \in L^{2}(\Omega)$. For the first equation in (3.5), by taking $\boldsymbol{\psi}=\boldsymbol{v}$, we obtain

$$
\begin{aligned}
\left\|\nabla \boldsymbol{v}^{t}\right\|_{0, \Omega}^{2}=<\mathbf{f}, \boldsymbol{v}>-<\nabla \mathbf{s}^{t}, \nabla \boldsymbol{v}^{t}>-\operatorname{Re}\left(<\left(\nabla \boldsymbol{v}^{t}\right)^{t} \mathbf{u}+\mathbf{U}^{t} \boldsymbol{v}, \boldsymbol{v}>+<\left(\nabla \mathbf{s}^{t}\right)^{t} \mathbf{u}+\mathbf{U}^{t} \mathbf{s}, \boldsymbol{v}>\right) \\
\leq|<\mathbf{f}, \boldsymbol{v}>|+\left|<\nabla \mathbf{s}^{t}, \nabla \boldsymbol{v}^{t}>\right| \\
\quad+\operatorname{Re}\left|<\mathbf{U}^{t} \boldsymbol{v}, \boldsymbol{v}>+<\left(\nabla \boldsymbol{v}^{t}\right)^{t} \mathbf{u}, \boldsymbol{v}>+<\mathbf{U}^{t} \mathbf{s}, \boldsymbol{v}>+<\left(\nabla \mathbf{s}^{t}\right)^{t} \mathbf{u}, \boldsymbol{v}>\right|
\end{aligned}
$$

For the first term of the upper bound in (3.6), recall the definition of the $H^{-1}(\Omega)$ norm:

$$
\|\mathbf{f}\|_{-1, \Omega}:=\sup _{0 \neq \boldsymbol{v} \in H_{0}^{1}(\Omega)} \frac{<\mathbf{f}, \boldsymbol{v}>}{|\boldsymbol{v}|_{1, \Omega}} \Rightarrow \frac{<\mathbf{f}, \boldsymbol{v}>}{|\boldsymbol{v}|_{1, \Omega}} \leq\|\mathbf{f}\|_{-1, \Omega}, \quad \forall \boldsymbol{v} \neq 0 \in H_{0}^{1}(\Omega)^{n} .
$$

Hence, for all $\boldsymbol{v} \neq 0 \in H_{0}^{1}(\Omega)^{n}$, we have $<\mathbf{f}, \boldsymbol{v}>\leq\|\mathbf{f}\|_{-1, \Omega}\|\nabla \boldsymbol{v}\|_{0, \Omega}$. To bound the second term, we use the Cauchy-Schwarz's inequality:

$$
<\nabla \mathbf{s}^{t}, \nabla \boldsymbol{v}^{t}>\leq\left\|\nabla \mathbf{s}^{t}\right\|_{0, \Omega}\left\|\nabla \boldsymbol{v}^{t}\right\|_{0, \Omega}=|\mathbf{s}|_{1, \Omega}\left\|\nabla \boldsymbol{v}^{t}\right\|_{0, \Omega} \stackrel{(3.4)}{\leq} C\|g\|_{0, \Omega}\left\|\nabla \boldsymbol{v}^{t}\right\|_{0, \Omega} .
$$

It remains to derive bounds for the last four terms, which are classified in [5] as trilinear. In the following, $C$ denotes a generic constant that might depend on $R e, \Omega,\|\mathbf{u}\|_{1}$, and $\|\mathbf{U}\|_{1}$.

According to the Sobolev Imbedding Theorem I.1.3 in [5], the space $H^{1}(\Omega)$ is continuously imbedded in $L^{4}(\Omega)$ for $n \leq 4$. Then,

$$
\begin{aligned}
|<\mathbf{U} \boldsymbol{v}, \boldsymbol{v}>| & =\left|\sum_{i, j=1}^{n} \int_{\Omega} v_{j} U_{i j} v_{i} d x\right| \leq \sum_{i, j=1}^{n}\left\|v_{j}\right\|_{0, \Omega}\left\|U_{i j}\right\|_{0,4, \Omega}\left\|v_{i}\right\|_{0,4, \Omega} \\
& \leq C\|\boldsymbol{v}\|_{0, \Omega}\|\mathbf{U}\|_{1, \Omega}\|\boldsymbol{v}\|_{1, \Omega} \leq C\|\boldsymbol{v}\|_{\delta_{\mathrm{o}}+\delta, \Omega}\|\mathbf{U}\|_{1, \Omega}|\boldsymbol{v}|_{1, \Omega} .
\end{aligned}
$$

The last inequality is a result of the Poincaré-Friedrichs inequality $\left(\|\boldsymbol{v}\|_{1, \Omega} \leq C|\boldsymbol{v}|_{1, \Omega}\right)$. Applying the Sobolev Imbedding Theorem to the second trilinear term leads to

$$
<\left(\nabla \boldsymbol{v}^{t}\right)^{t} \mathbf{u}, \boldsymbol{v}>\leq\left\|\nabla \boldsymbol{v}^{t}\right\|_{0, \Omega}\left\|\mathbf{u}^{t} \boldsymbol{v}\right\|_{0, \Omega} \leq\left\|\nabla \boldsymbol{v}^{t}\right\|_{0, \Omega}\|\mathbf{u}\|_{1, \Omega}\|\boldsymbol{v}\|_{\delta_{o}+\delta, \Omega} .
$$

Similar arguments hold for the remaining two trilinear terms. Hence,

$$
<\mathbf{U}^{t} \mathbf{s}, \boldsymbol{v}>\leq C\|\mathbf{U}\|_{1, \Omega}|\mathbf{s}|_{1, \Omega}\|\boldsymbol{v}\|_{1, \Omega} \leq C\|\mathbf{U}\|_{1, \Omega}\left\|\nabla \boldsymbol{v}^{t}\right\|_{0, \Omega}\|g\|_{0, \Omega}
$$

and

$$
<\left(\nabla \mathbf{s}^{t}\right)^{t} \mathbf{u}, \boldsymbol{v}>\leq C|\mathbf{s}|_{1, \Omega}\|\mathbf{u}\|_{1, \Omega}\|\boldsymbol{v}\|_{1, \Omega} \leq C\|\mathbf{u}\|_{1, \Omega}\left\|\nabla \boldsymbol{v}^{t}\right\|_{0, \Omega}\|g\|_{0, \Omega} .
$$

Combining the results yields

$$
\begin{gathered}
\left\|\nabla \boldsymbol{v}^{t}\right\|_{0, \Omega}^{2} \leq\|\mathbf{f}\|_{-1, \Omega}\|\nabla \boldsymbol{v}\|_{0, \Omega}+C\|g\|_{0, \Omega}\|\nabla \boldsymbol{v}\|_{0, \Omega}+C\|\mathbf{U}\|_{1, \Omega}\|\boldsymbol{v}\|_{\delta_{0}+\delta, \Omega}\|\nabla \boldsymbol{v}\|_{0, \Omega} \\
+C\left(\|\mathbf{U}\|_{1, \Omega}+\|\mathbf{u}\|_{1, \Omega}\right)\|g\|_{0, \Omega}\|\nabla \boldsymbol{v}\|_{0, \Omega} .
\end{gathered}
$$


Canceling $\|\nabla \boldsymbol{v}\|_{0, \Omega}$ gives

$$
\left\|\nabla \boldsymbol{v}^{t}\right\|_{0, \Omega} \leq C\left(R e,\|\mathbf{u}\|_{1, \Omega},\|\mathbf{U}\|_{1, \Omega}, \Omega\right)\left[\|\mathbf{f}\|_{-1, \Omega}+\|g\|_{0, \Omega}+\|\boldsymbol{v}\|_{\delta_{\mathrm{o}}+\delta, \Omega}\right] .
$$

To bound $q$, choose $\boldsymbol{\psi} \in H_{0}^{1}(\Omega)^{n}$ according to Lemma 4.1 in [7] such that

$$
\nabla \cdot \boldsymbol{\psi}=q \quad \text { and } \quad|\boldsymbol{\psi}|_{1, \Omega} \leq C\|q\|_{0, \Omega} .
$$

Using again the first equation of (3.5), we obtain

$$
\begin{aligned}
&\|q\|_{0, \Omega}^{2}=<\nabla \boldsymbol{v}^{t}, \nabla \boldsymbol{\psi}^{t}>+R e<\left(\nabla \boldsymbol{v}^{t}\right)^{t} \mathbf{u}+\mathbf{U}^{t} \boldsymbol{v}, \boldsymbol{\psi}> \\
&-<\mathbf{f}, \boldsymbol{\psi}>+<\nabla \mathbf{s}^{t}, \nabla \boldsymbol{\psi}^{t}>+R e<\left(\nabla \mathbf{s}^{t}\right)^{t} \mathbf{u}+\mathbf{U}^{t} \mathbf{s}, \boldsymbol{\psi}> \\
& \leq<\nabla \boldsymbol{v}^{t}, \nabla \boldsymbol{\psi}^{t}>+R e<\left(\nabla \boldsymbol{v}^{t}\right)^{t} \mathbf{u}+\mathbf{U}^{t} \boldsymbol{v}, \boldsymbol{\psi}> \\
&+|<\mathbf{f}, \boldsymbol{\psi}>|+\left\langle\nabla \mathbf{s}^{t}, \nabla \boldsymbol{\psi}^{t}>+\operatorname{Re}<\left(\nabla \mathbf{s}^{t}\right)^{t} \mathbf{u}+\mathbf{U}^{t} \mathbf{s}, \boldsymbol{\psi}>.\right.
\end{aligned}
$$

We proceed similarly as with the bound $\left\|\nabla \boldsymbol{v}^{t}\right\|_{0, \Omega}$. For all $\boldsymbol{\psi} \neq 0 \in H_{0}^{1}(\Omega)^{n}$,

$$
\begin{gathered}
<\mathbf{f}, \boldsymbol{\psi}>\leq\|\mathbf{f}\|_{-1, \Omega}|\boldsymbol{\psi}|_{1, \Omega} \stackrel{(3.12)}{\leq} C\|\mathbf{f}\|_{-1, \Omega}\|q\|_{0, \Omega}, \\
<\nabla \boldsymbol{v}^{t}, \nabla \boldsymbol{\psi}^{t}>\leq\left\|\nabla \boldsymbol{v}^{t}\right\|_{0, \Omega}\left\|\nabla \boldsymbol{\psi}^{t}\right\|_{0, \Omega}=|\boldsymbol{v}|_{1, \Omega}|\boldsymbol{\psi}|_{1, \Omega} \stackrel{(3.12)}{\leq} C|\boldsymbol{v}|_{1, \Omega}\|q\|_{0, \Omega},
\end{gathered}
$$

and

$$
<\nabla \mathbf{s}^{t}, \nabla \boldsymbol{\psi}^{t}>\leq\left\|\nabla \mathbf{s}^{t}\right\|_{0, \Omega}\left\|\nabla \boldsymbol{\psi}^{t}\right\|_{0, \Omega}=|\mathbf{s}|_{1, \Omega}|\boldsymbol{\psi}|_{1, \Omega} \stackrel{(3.12)}{\leq} C|\mathbf{s}|_{1, \Omega}\|q\|_{0, \Omega} .
$$

The bounds for trilinear terms $\left\langle\left(\nabla \boldsymbol{v}^{t}\right)^{t} \mathbf{u}, \boldsymbol{\psi}>\right.$ and $\left\langle\left(\nabla \mathbf{s}^{t}\right)^{t} \mathbf{u}, \boldsymbol{\psi}\right\rangle$ follow directly by applying Lemma IV.2.1 in [5] and the Poincaré-Friedrichs inequality:

$$
<\left(\nabla \boldsymbol{v}^{t}\right)^{t} \mathbf{u}, \boldsymbol{\psi}>\leq C|\boldsymbol{v}|_{1, \Omega}\|\mathbf{u}\|_{1, \Omega}\|\boldsymbol{\psi}\|_{1, \Omega} \leq C\|\mathbf{u}\|_{1, \Omega}\left\|\nabla \boldsymbol{v}^{t}\right\|_{0, \Omega}|\boldsymbol{\psi}|_{1, \Omega}
$$

and

$$
<\left(\nabla \mathbf{s}^{t}\right)^{t} \mathbf{u}, \boldsymbol{\psi}>\leq C|\mathbf{s}|_{1, \Omega}\|\mathbf{u}\|_{1, \Omega}\|\boldsymbol{\psi}\|_{1, \Omega} \leq C\|\mathbf{u}\|_{1, \Omega}|\mathbf{s}|_{1, \Omega}|\boldsymbol{\psi}|_{1, \Omega} .
$$

For the remaining trilinear terms, we follow the argument in (3.7):

$$
<\mathbf{U}^{t} \boldsymbol{v}, \boldsymbol{\psi}>\leq C\|\mathbf{U}\|_{1, \Omega}\|\boldsymbol{v}\|_{1, \Omega}|\boldsymbol{\psi}|_{1, \Omega} \leq C\|\mathbf{U}\|_{1, \Omega}\left\|\nabla \boldsymbol{v}^{t}\right\|_{0, \Omega}|\boldsymbol{\psi}|_{1, \Omega}
$$

and

$$
<\mathbf{U}^{t} \mathbf{s}, \boldsymbol{\psi}>\leq C\|\mathbf{U}\|_{1, \Omega}\|\mathbf{s}\|_{1, \Omega}|\boldsymbol{\psi}|_{1, \Omega} \leq C\|\mathbf{U}\|_{1, \Omega}|\mathbf{s}|_{1, \Omega}|\boldsymbol{\psi}|_{1, \Omega} .
$$

With (3.13)-(3.19), we have

$$
\begin{gathered}
\|q\|_{0, \Omega}^{2} \leq \quad C\|\mathbf{f}\|_{-1, \Omega}\|q\|_{0, \Omega}+C|\boldsymbol{v}|_{1, \Omega}\|q\|_{0, \Omega}+C|\mathbf{s}|_{1, \Omega}\|q\|_{0, \Omega} \\
+C\left(\left\|\nabla \boldsymbol{v}^{t}\right\|_{0, \Omega}+|\mathbf{s}|_{1, \Omega}\right)|\boldsymbol{\psi}|_{1, \Omega} \\
\stackrel{(3.12)}{\leq} C\left(R e,\|\mathbf{u}\|_{1, \Omega},\|\mathbf{U}\|_{1, \Omega}, \Omega\right)\left(\|\mathbf{f}\|_{-1, \Omega}+\left\|\nabla \boldsymbol{v}^{t}\right\|_{0, \Omega}+|\mathbf{s}|_{1, \Omega}\right)\|q\|_{0, \Omega} \\
\stackrel{(3.4),(3.11)}{\leq} C\left(R e,\|\mathbf{u}\|_{1, \Omega},\|\mathbf{U}\|_{1, \Omega}, \Omega\right)\left(\|\mathbf{f}\|_{-1, \Omega}+\|g\|_{0, \Omega}+\|\boldsymbol{v}\|_{\delta_{0}+\delta, \Omega}\right)\|q\|_{0, \Omega} .
\end{gathered}
$$


Canceling $\|q\|_{0, \Omega}$ results in

$$
\|q\|_{0, \Omega} \leq C\left(R e,\|\mathbf{u}\|_{1, \Omega},\|\mathbf{U}\|_{1, \Omega}, \Omega\right)\left(\|\mathbf{f}\|_{-1, \Omega}+\|g\|_{0, \Omega}+\|\boldsymbol{v}\|_{\delta_{\mathrm{o}}+\delta, \Omega}\right) .
$$

Recall that we seek an estimate for $\left\|\nabla \mathbf{v}^{t}\right\|_{0, \Omega}+\|q\|_{0, \Omega}$ in terms of $\mathbf{v}$ and $q$ and not for $\left\|\nabla \boldsymbol{v}^{t}\right\|_{0, \Omega}+\|q\|_{0, \Omega}$ in terms of $\boldsymbol{v}$ and $q$. Earlier, we defined $\boldsymbol{v}$ to be the difference between $\mathbf{v}$ and $\mathbf{s}$. Now, adding $\mathbf{s}$ to $\boldsymbol{v}$ leads to estimates for $\left\|\nabla \mathbf{v}^{t}\right\|_{0, \Omega}+\|q\|_{0, \Omega}$ in terms of $\mathbf{v}$ and $q$ :

$$
\begin{aligned}
\left\|\nabla \mathbf{v}^{t}\right\|_{0, \Omega} & \leq\left\|\nabla \boldsymbol{v}^{t}+\nabla \mathbf{s}^{t}\right\|_{0, \Omega} \leq\left\|\nabla \boldsymbol{v}^{t}\right\|_{0, \Omega}+\left\|\nabla \mathbf{s}^{t}\right\|_{0, \Omega} \\
& \stackrel{(3.11)}{\leq} C\left(R e,\|\mathbf{u}\|_{1, \Omega},\|\mathbf{U}\|_{1, \Omega}, \Omega\right)\left[\|\mathbf{f}\|_{-1, \Omega}+\|g\|_{0, \Omega}+\|\boldsymbol{v}\|_{\delta_{0}+\delta, \Omega}+\left\|\nabla \mathbf{s}^{t}\right\|_{0, \Omega}\right] \\
& \leq C\left(R e,\|\mathbf{u}\|_{1, \Omega},\|\mathbf{U}\|_{1, \Omega}, \Omega\right)\left[\|\mathbf{f}\|_{-1, \Omega}+\|g\|_{0, \Omega}+\|\mathbf{v}\|_{\delta_{0}+\delta, \Omega}+C|\mathbf{s}|_{1, \Omega}\right] \\
& \stackrel{(3.4)}{\leq} C\left(R e,\|\mathbf{u}\|_{1, \Omega},\|\mathbf{U}\|_{1, \Omega}, \Omega\right)\left[\|\mathbf{f}\|_{-1, \Omega}+\|g\|_{0, \Omega}+\|\mathbf{v}\|_{\delta_{0}+\delta, \Omega}+C\|g\|_{0, \Omega}\right] \\
& \leq C\left(R e,\|\mathbf{u}\|_{1, \Omega},\|\mathbf{U}\|_{1, \Omega}, \Omega\right)\left[\|\mathbf{f}\|_{-1, \Omega}+\|g\|_{0, \Omega}+\|\mathbf{v}\|_{\delta_{0}+\delta, \Omega}\right] .
\end{aligned}
$$

Similarly,

$$
\begin{aligned}
\|q\|_{0, \Omega} & \leq C\left(R e,\|\mathbf{u}\|_{1, \Omega},\|\mathbf{U}\|_{1, \Omega}, \Omega\right)\left[\|\mathbf{f}\|_{-1, \Omega}+\|g\|_{0, \Omega}+\|\boldsymbol{v}\|_{\delta_{o}+\delta, \Omega}\right] \\
& \leq C\left(R e,\|\mathbf{u}\|_{1, \Omega},\|\mathbf{U}\|_{1, \Omega}, \Omega\right)\left[\|\mathbf{f}\|_{-1, \Omega}+\|g\|_{0, \Omega}+\|\mathbf{v}\|_{\delta_{0}+\delta, \Omega}\right]
\end{aligned}
$$

Combining the bounds for $\left\|\nabla \mathbf{v}^{t}\right\|_{0, \Omega}$ and $\|q\|_{0, \Omega}$ results in the a priori estimate

$$
\left\|\nabla \mathbf{v}^{t}\right\|_{0, \Omega}+\|q\|_{0, \Omega} \leq C\left(R e,\|\mathbf{u}\|_{1, \Omega},\|\mathbf{U}\|_{1, \Omega}, \Omega\right)\left[\|\mathbf{f}\|_{-1, \Omega}+\|g\|_{0, \Omega}+\|\mathbf{v}\|_{\delta_{0}+\delta, \Omega}\right] .
$$

Theorem 4.1 in [7] removes the $\|\mathbf{v}\|_{\delta_{0}+\delta, \Omega}$ term by assuming uniqueness of the solution, $(\mathbf{v}, \mathbf{V}, q) \in \mathcal{V}$. This is a direct consequence of the standard compactness argument. Since $H_{0}^{1}(\Omega)$ is compact in $H^{\delta_{\mathrm{o}}+\delta}(\Omega)$, where $\delta \in\left(0, \frac{1}{2}\right)$ and $\delta_{\mathrm{o}}=0$ or $\frac{1}{2}$ depending on the spatial dimension of the domain, we can apply the standard compactness argument to (3.21) in a way similar to the estimate $\left\|\nabla \mathbf{v}^{t}\right\|_{0, \Omega}+\|q\|_{0, \Omega} \leq C(R e, \mathbf{u}, \Omega)\left[\|\mathbf{f}\|_{-1, \Omega}+\right.$ $\left.\|g\|_{0, \Omega}+\|\mathbf{v}\|_{0, \Omega}\right]$ in the proof of Theorem 4.1 of [7]. We also note that the slightly different constant in (3.21) (compared to the regularity estimate in [7]) has no further implications in [7] on Theorems 3.2, 4.1, and 4.2 or their proofs. Thus, we obtain continuity and coercivity for $\mathcal{L}^{\prime}(\mathbf{x})[\mathbf{y}]$ under the somewhat weaker assumptions of $\mathbf{x}$ and $\mathbf{y}$ being in $\mathcal{V}$.

We conclude that there exist two positive constants, $\tilde{c_{3}}$ and $\tilde{C_{3}}$, which depend on $R e$, the $H^{1}$-norm of $\mathbf{u}$ and $\mathbf{U}$, and $\Omega$, such that

$$
\tilde{c_{3}}(\mathbf{x})\|\mathbf{y}\|_{1, \Omega}^{2} \leq\left\|\mathcal{L}^{\prime}(\mathbf{x})[\mathbf{y}]\right\|_{0, \Omega}^{2} \leq \tilde{C_{3}}(\mathbf{x})\|\mathbf{y}\|_{1, \Omega}^{2},
$$

for all $\mathbf{y} \in \mathcal{V}$.

The next lemma establishes, for all $\mathbf{x} \in \overline{\mathcal{B}}\left(\mathbf{x}_{*}, r\right)$ and $r$ sufficiently small, a uniform coercivity and continuity bound on $\left\|\mathcal{L}^{\prime}(\mathbf{x})[\mathbf{y}]\right\|_{0, \Omega}^{2}$. 
LEMma 3.3. Let $\mathbf{x}_{*}$ be an isolated solution of $(2.2)$ and let $\tilde{c}_{3}\left(\mathbf{x}_{*}\right)$ and $\tilde{C}_{3}\left(\mathbf{x}_{*}\right)$ be the respective coercivity and continuity constants as defined in Lemma 3.2. Then,

$$
c_{3}\|\mathbf{y}\|_{1, \Omega}^{2} \leq\left\|\mathcal{L}^{\prime}(\mathbf{x})[\mathbf{y}]\right\|_{0, \Omega}^{2} \leq C_{3}\|\mathbf{y}\|_{1, \Omega}^{2},
$$

for all $\mathbf{x}=(\mathbf{u}, \mathbf{U}, p) \in \overline{\mathcal{B}}\left(\mathbf{x}_{*}, r\right), \mathbf{y}=(\mathbf{v}, \mathbf{V}, q)$, and $\mathbf{z}=(\mathbf{w}, \mathbf{W}, t) \in \mathcal{V}$, where $c_{3}:=$ $\tilde{c}_{3}\left(\mathbf{x}_{*}\right)-\gamma_{0} r^{2}>0$ provided $r<\sqrt{\tilde{c}_{3}\left(\mathbf{x}_{*}\right) / \gamma_{0}}$ and $C_{3}:=\tilde{C}_{3}\left(\mathbf{x}_{*}\right)+\gamma_{0} r^{2}>0$.

Proof. For all $\mathbf{x}, \mathbf{y} \in \overline{\mathcal{B}}\left(\mathbf{x}_{*}, r\right)$ and $r$ determined later, Lemma 3.2 implies that

$$
\begin{aligned}
\left\|\mathcal{L}^{\prime}(\mathbf{x})[\mathbf{y}]\right\|_{0, \Omega}^{2} & =\left\|\mathcal{L}^{\prime}(\mathbf{x})[\mathbf{y}]-\mathcal{L}^{\prime}\left(\mathbf{x}_{*}\right)[\mathbf{y}]+\mathcal{L}^{\prime}\left(\mathbf{x}_{*}\right)[\mathbf{y}]\right\|_{0, \Omega}^{2} \\
& \leq\left\|\mathcal{L}^{\prime \prime}(\tilde{\mathbf{x}})\left[\mathbf{x}-\mathbf{x}_{*}, \mathbf{y}\right]\right\|_{0, \Omega}^{2}+\left\|\mathcal{L}^{\prime}\left(\mathbf{x}_{*}\right)[\mathbf{y}]\right\|_{0, \Omega}^{2} \\
& \leq\left(\gamma_{0}\left\|\mathbf{x}-\mathbf{x}_{*}\right\|_{1, \Omega}^{2}+\tilde{C}_{3}\left(\mathbf{x}_{*}\right)\right)\|\mathbf{y}\|_{1, \Omega}^{2} \\
& \leq\left(\gamma_{0} r^{2}+\tilde{C}_{3}\left(\mathbf{x}_{*}\right)\right)\|\mathbf{y}\|_{1, \Omega}^{2}=: C_{3}\|\mathbf{y}\|_{1, \Omega}^{2}
\end{aligned}
$$

and

$$
\begin{aligned}
\left\|\mathcal{L}^{\prime}(\mathbf{x})[\mathbf{y}]\right\|_{0, \Omega}^{2} & =\left\|\mathcal{L}^{\prime}(\mathbf{x})[\mathbf{y}]-\mathcal{L}^{\prime}\left(\mathbf{x}_{*}\right)[\mathbf{y}]+\mathcal{L}^{\prime}\left(\mathbf{x}_{*}\right)[\mathbf{y}]\right\|_{0, \Omega}^{2} \\
& \geq-\left\|\mathcal{L}^{\prime \prime}(\tilde{\mathbf{x}})\left[\mathbf{x}-\mathbf{x}_{*}, \mathbf{y}\right]\right\|_{0, \Omega}^{2}+\left\|\mathcal{L}^{\prime}\left(\mathbf{x}_{*}\right)[\mathbf{y}]\right\|_{0, \Omega}^{2} \\
& \geq\left(-\gamma_{0}\left\|\mathbf{x}-\mathbf{x}_{*}\right\|_{1, \Omega}^{2}+\tilde{c_{3}}\left(\mathbf{x}_{*}\right)\right)\|\mathbf{y}\|_{1, \Omega}^{2} \\
& \geq\left(-\gamma_{0} r^{2}+\tilde{c_{3}}\left(\mathbf{x}_{*}\right)\right)\|\mathbf{y}\|_{1, \Omega}^{2}=: \quad c_{3}\|\mathbf{y}\|_{1, \Omega}^{2} .
\end{aligned}
$$

Constant $C_{3}$ is obviously positive and $r<\sqrt{\frac{\tilde{c}\left(\mathbf{x}_{*}\right)}{\gamma_{0}}}$ ensures that $c_{3}$ is positive.

Note that $c_{3}$ and $C_{3}$ in Lemma 3.3 depend only on $R e, r$, and $\Omega$.

Next, we derive continuity and coercivity results for the full nonquadratic functional, $\mathcal{F}(\mathbf{x} ; \mathbf{g})$. First, we establish these results for its second Fréchet derivative. Then, almost as a direct implication of this, we achieve continuity and coercivity for the functional norm itself. We restrict ourselves to an $r$ that is small enough to ensure that all of these results hold uniformly for $\mathbf{x} \in \overline{\mathcal{B}}\left(\mathbf{x}_{*}, r\right)$.

TheOREM 1. There exist an $r>0$ such that, for any $\mathbf{x} \in \overline{\mathcal{B}}\left(\mathbf{x}_{*}, r\right)$, the second Fréchet derivative of $\mathcal{F}(\mathbf{x} ; \mathbf{g})$ in direction $[\mathbf{y}, \mathbf{y}], \mathbf{y} \in \mathcal{V}$, is positive. Furthermore, there exist two positive constants, $c_{4}$ and $C_{4}$, which depend only on Re, $r$, and $\Omega$, such that

$$
\mathcal{F}^{\prime \prime}(\mathbf{x} ; \mathbf{g})[\mathbf{y}, \mathbf{z}] \leq C_{4}\|\mathbf{y}\|_{1, \Omega}\|\mathbf{z}\|_{1, \Omega}
$$

and

$$
c_{4}\|\mathbf{y}\|_{1, \Omega}^{2} \leq \mathcal{F}^{\prime \prime}(\mathbf{x} ; \mathbf{g})[\mathbf{y}, \mathbf{y}]
$$

for any $\mathbf{x} \in \overline{\mathcal{B}}\left(\mathbf{x}_{*}, r\right)$ and all $\mathbf{y} \in \mathcal{V}$.

Proof. First, we show that $\mathcal{F}^{\prime \prime}(\mathbf{x} ; \mathbf{g})[\mathbf{y}, \mathbf{y}]$ is positive. Let $\mathbf{x} \in \overline{\mathcal{B}}\left(\mathbf{x}_{*}, r\right)$, with $r<\sqrt{\frac{c \tilde{c}_{3}\left(\mathbf{x}_{*}\right)}{\gamma_{0}}}$, as in the proof of Lemma 3.3. Then the Cauchy-Schwarz inequality and Lemmas 3.1 and 3.3 show that

$$
\begin{aligned}
<\mathcal{L}(\mathbf{x})-g, \mathcal{L}^{\prime \prime}(\mathbf{x})[\mathbf{y}, \mathbf{y}]> & =<\mathcal{L}^{\prime}(\tilde{\mathbf{x}})\left[\mathbf{x}-\mathbf{x}_{*}\right], \mathcal{L}^{\prime \prime}(\mathbf{x})[\mathbf{y}, \mathbf{y}]> \\
& \leq\left\|\mathcal{L}^{\prime}(\tilde{\mathbf{x}})\left[\mathbf{x}-\mathbf{x}_{*}\right]\right\|_{0, \Omega} \cdot\left\|\mathcal{L}^{\prime \prime}(\mathbf{x})[\mathbf{y}, \mathbf{y}]\right\|_{0, \Omega} \\
& \leq \sqrt{C_{3}} \gamma_{0}\left\|\mathbf{x}-\mathbf{x}_{*}\right\|_{1, \Omega}\|\mathbf{y}\|_{1, \Omega}^{2}
\end{aligned}
$$


where $\tilde{\mathbf{x}} \in\left[\mathbf{x}_{*}, \mathbf{x}\right] \subset \overline{\mathcal{B}}\left(\mathbf{x}_{*}, r\right)$. Then, by (2.4), (3.24), and Lemma 3.3, we have

$$
\begin{aligned}
\mathcal{F}^{\prime \prime}(\mathbf{x} ; \mathrm{g})[\mathbf{y}, \mathbf{y}] & =2\left\|\mathcal{L}^{\prime}(\mathbf{x})[\mathbf{y}]\right\|_{0, \Omega}^{2}+2<\mathcal{L}(\mathbf{x})-\mathrm{g}, \mathcal{L}^{\prime \prime}(\mathbf{x})[\mathbf{y}, \mathbf{y}]> \\
& \geq 2 c_{3}\|\mathbf{y}\|_{1, \Omega}^{2}-2 \sqrt{C_{3}} \gamma_{0}\left\|\mathbf{x}-\mathbf{x}_{*}\right\|_{1, \Omega}\|\mathbf{y}\|_{1, \Omega}^{2} \\
& =\left(2 c_{3}-2 \sqrt{C_{3}} \gamma_{0}\left\|\mathbf{x}-\mathbf{x}_{*}\right\|_{1, \Omega}\right)\|\mathbf{y}\|_{1, \Omega}^{2} \\
& =\left(2 \tilde{c}_{3}\left(\mathbf{x}_{*}\right)-2 \gamma_{0} r^{2}-2 \gamma_{0} r \sqrt{\tilde{C}_{3}\left(\mathbf{x}_{*}\right)+\gamma_{0} r^{2}}\right)\|\mathbf{y}\|_{1, \Omega}^{2} \\
& =: c_{4}(r)\|\mathbf{y}\|_{1, \Omega}^{2} .
\end{aligned}
$$

Since $c_{4}(r)$ is continuous with respect to $r$ and $c_{4}(0)=2 \tilde{c}_{3}\left(\mathbf{x}_{*}\right)>0$, then $c_{4}(r)$ is positive for small enough $r>0$. This $r$ ensures that the second Fréchet derivative of $\mathcal{F}(\mathbf{x} ; \mathrm{g})$ in direction $[\mathbf{y}, \mathbf{y}]$ is positive for all $\mathbf{x} \in \overline{\mathcal{B}}\left(\mathbf{x}_{*}, r\right)$.

The upper bound for $\mathcal{F}^{\prime \prime}(\mathbf{x} ; \mathrm{g})[\mathbf{y}, \mathbf{z}]$ follows by Lemma 3.3, (3.24), and Lemma 3.1:

$$
\begin{aligned}
\mathcal{F}^{\prime \prime}(\mathbf{x} ; \mathrm{g})[\mathbf{y}, \mathbf{z}] & =2\left\|\mathcal{L}^{\prime}(\mathbf{x})[\mathbf{y}]\right\|_{0, \Omega}\left\|\mathcal{L}^{\prime}(\mathbf{x})[\mathbf{z}]\right\|_{0, \Omega}+2<\mathcal{L}(\mathbf{x})-\mathrm{g}, \mathcal{L}^{\prime \prime}(\mathbf{x})[\mathbf{y}, \mathbf{z}]> \\
& \leq 2 C_{3}\|\mathbf{y}\|_{1, \Omega}\|\mathbf{z}\|_{1, \Omega}+2 \sqrt{C_{3}} \gamma_{0}\left\|\mathbf{x}-\mathbf{x}_{*}\right\|_{1, \Omega}\|\mathbf{y}\|_{1, \Omega}\|\mathbf{z}\|_{1, \Omega} \\
& \leq\left(2 C_{3}+2 \sqrt{C_{3}} \gamma_{0} r\right)\|\mathbf{y}\|_{1, \Omega}\|\mathbf{z}\|_{1, \Omega} \\
& =: C_{4}(r)\|\mathbf{y}\|_{1, \Omega}\|\mathbf{z}\|_{1, \Omega} .
\end{aligned}
$$

REMARK 3. We henceforth assume that the $r$ of Theorem 1 is so small that it is less than $0.4 \frac{c_{3}}{\sqrt{C_{3}} \gamma_{0}}$. This can be always arranged by choosing $r$ small enough.

REMARK 4. The results of Lemma 3.1, Lemma 3.3, and Theorem 1 still hold if we restrict ourselves to a subspace of $\mathcal{V}$ by assuming that $\mathbf{x}=\mathbf{x}^{h} \in \overline{\mathcal{B}}^{h}\left(\mathbf{x}_{*}, r\right)$, $\mathbf{y}=\mathbf{y}^{h} \in \mathcal{S}^{h}$, and $\mathbf{z}=\mathbf{z}^{h} \in \mathcal{S}^{h}$.

THEOREM 2. The nonquadratic functional, $\mathcal{F}(\mathbf{x} ; \mathrm{g})$, is coercive and continuous for all $\mathbf{x} \in \overline{\mathcal{B}}\left(\mathbf{x}_{*}, r\right)$, where $r, c_{3}$, and $C_{3}$ are defined as in Theorem 1:

$$
\frac{1}{2} c_{3}\left\|\mathbf{x}-\mathbf{x}_{*}\right\|_{1, \Omega}^{2} \leq \mathcal{F}(\mathbf{x} ; \mathbf{g}) \leq \frac{1}{2} C_{3}\left\|\mathbf{x}-\mathbf{x}_{*}\right\|_{1, \Omega}^{2} .
$$

Proof. For any $\mathbf{x} \in \overline{\mathcal{B}}\left(\mathbf{x}_{*}, r\right)$ and some $\tilde{\mathbf{x}} \in\left[\mathbf{x}_{*}, \mathbf{x}\right] \subset \overline{\mathcal{B}}\left(\mathbf{x}_{*}, r\right)$, we have

$$
\mathcal{F}(\mathbf{x} ; \mathbf{g})=\mathcal{F}\left(\mathbf{x}_{*} ; \mathrm{g}\right)+\mathcal{F}^{\prime}\left(\mathbf{x}_{*} ; \mathrm{g}\right)\left[\mathbf{x}-\mathbf{x}_{*}\right]+\frac{1}{2} \mathcal{F}^{\prime \prime}(\tilde{\mathbf{x}} ; \mathrm{g})\left[\mathbf{x}-\mathbf{x}_{*}, \mathbf{x}-\mathbf{x}_{*}\right]
$$

The result now follows from the fact that $\mathcal{F}\left(\mathbf{x}_{*} ; \mathbf{g}\right)=\mathcal{F}^{\prime}\left(\mathbf{x}_{*} ; \mathbf{g}\right)\left[\mathbf{x}-\mathbf{x}_{*}\right]=0$ (see $(2.3)$ for the second equality) and Theorem 1.

A similar result can be easily established for discrete space $\mathcal{S}^{h}$.

THEOREM 3. The functional norm, $\sqrt{\mathcal{F}\left(\mathrm{x}^{h} ; \mathrm{g}\right)-\mathcal{F}\left(\mathbf{x}_{*}^{h} ; \mathrm{g}\right)}$, is coercive and continuous for all $\mathbf{x}^{h} \in \overline{\mathcal{B}}^{h}\left(\mathbf{x}_{*}, r\right)$, where $r, c_{3}$, and $C_{3}$ are defined as in Theorem 1:

$$
\frac{1}{2} c_{3}\left\|\mathbf{x}^{h}-\mathbf{x}_{*}^{h}\right\|_{1, \Omega}^{2} \leq \mathcal{F}\left(\mathbf{x}^{h} ; \mathbf{g}\right)-\mathcal{F}\left(\mathbf{x}_{*}^{h} ; \mathbf{g}\right) \leq \frac{1}{2} C_{3}\left\|\mathbf{x}^{h}-\mathbf{x}_{*}^{h}\right\|_{1, \Omega}^{2} .
$$


Proof. For any $\mathbf{x}^{h} \in \overline{\mathcal{B}}^{h}\left(\mathbf{x}_{*}, r\right)$ and some $\tilde{\mathbf{x}} \in\left[\mathbf{x}_{*}, \mathbf{x}^{h}\right] \subset \overline{\mathcal{B}}^{h}\left(\mathbf{x}_{*}, r\right)$, we have

$$
\mathcal{F}\left(\mathbf{x}^{h} ; \mathrm{g}\right)-\mathcal{F}\left(\mathbf{x}_{*}^{h} ; \mathrm{g}\right)=\mathcal{F}^{\prime}\left(\mathbf{x}_{*}^{h} ; \mathrm{g}\right)\left[\mathbf{x}^{h}-\mathbf{x}_{*}^{h}\right]+\frac{1}{2} \mathcal{F}^{\prime \prime}(\tilde{\mathbf{x}} ; \mathrm{g})\left[\mathbf{x}^{h}-\mathbf{x}_{*}^{h}, \mathbf{x}^{h}-\mathbf{x}_{*}^{h}\right]
$$

From Definition 2.1, we know that $\mathcal{F}^{\prime}\left(\mathbf{x}_{*}^{h} ; \mathbf{g}\right)\left[\mathbf{x}^{h}-\mathbf{x}_{*}^{h}\right]=<\mathcal{L}\left(\mathbf{x}_{*}^{h}\right)-\mathrm{g}, \mathcal{L}^{\prime}\left(\mathbf{x}_{*}^{h}\right)\left[\mathbf{x}^{h}-\mathbf{x}_{*}^{h}\right]>=$ 0 , for all $\mathbf{x}^{h}-\mathbf{x}_{*}^{h} \in \mathcal{S}^{h}$ (see Remark 2). Hence, continuity and coercivity again follow directly from Theorem 1 .

4. Convergence. This section establishes unique minimizers of the functionals we use in $\overline{\mathcal{B}}\left(\mathbf{x}_{*}, r\right), \overline{\mathcal{B}}^{h}\left(\mathbf{x}_{*}, r\right)$, and $\overline{\mathcal{B}}^{2 h}\left(\mathbf{x}_{*}, r\right)$ under the assumption that $r$ and $h$ are sufficiently small. This is done in Lemmas 4.1, 4.2, and 4.3, respectively. Theorem 4 then shows that our coarse-grid correction and relaxation steps remain in a closed $H^{1}$-ball about $\mathbf{x}_{*}$ and it establishes uniform convergence of our two-level PML scheme.

LEMMA 4.1. Let $\mathbf{x}_{*}$ be an isolated solution of (2.2) and $r$ be defined as in Theorem 1. Then $\mathbf{x}_{*}$ is the unique minimizer in $\overline{\mathcal{B}}\left(\mathbf{x}_{*}, r\right)$ of $\mathcal{F}(\mathbf{x} ; \mathrm{g})$. It is characterized by $\mathcal{F}^{\prime}\left(\mathbf{x}_{*} ; \mathrm{g}\right)[\mathbf{y}]=\mathbf{0}$, for all $\mathbf{y} \in \mathcal{V}$, that is, it is the unique critical point in $\overline{\mathcal{B}}\left(\mathbf{x}_{*}, r\right)$.

Proof. The first assertion follows from (3.25). That $\mathbf{x}_{*}$ is a critical point follows from (2.3). We thus only need to show that it is the only critical point in $\overline{\mathcal{B}}\left(\mathbf{x}_{*}, r\right)$, that is, that $\mathcal{F}^{\prime}(\mathbf{x} ; \mathrm{g})[\mathbf{y}]=\mathbf{0}$ for all $\mathbf{y} \in \mathcal{V}$ and $\mathbf{x} \in \overline{\mathcal{B}}\left(\mathbf{x}_{*}, r\right)$ imply $\mathbf{x}=\mathbf{x}_{*}$. Under these assumptions, for all $\mathbf{y} \in \mathcal{V}$, we have

$$
0=\mathcal{F}^{\prime}(\mathbf{x} ; \mathbf{g})[\mathbf{y}]-\mathcal{F}^{\prime}\left(\mathbf{x}_{*} ; \mathrm{g}\right)[\mathbf{y}]=\mathcal{F}^{\prime \prime}(\tilde{\mathbf{x}} ; \mathrm{g})\left[\mathbf{y}, \mathbf{x}-\mathbf{x}_{*}\right]
$$

for some $\tilde{\mathbf{x}} \in\left[\mathbf{x}_{*}, \mathbf{x}\right] \subset \overline{\mathcal{B}}\left(\mathbf{x}_{*}, r\right)$. With $\mathbf{y}=\mathbf{x}-\mathbf{x}_{*} \in \mathcal{V}$ and Theorem 1 , we thus obtain

$$
0=\mathcal{F}^{\prime \prime}(\tilde{\mathbf{x}} ; \mathbf{g})\left[\mathbf{x}-\mathbf{x}_{*}, \mathbf{x}-\mathbf{x}_{*}\right] \geq c_{4}\left\|\mathbf{x}-\mathbf{x}_{*}\right\|_{1, \Omega}^{2}
$$

Therefore, $\mathbf{x}=\mathbf{x}_{*}$ and the proof is complete.

Next, we prove the discrete analog to Lemma 4.1.

LEMмA 4.2. Let $\mathbf{x}_{*}$ be an isolated solution of (2.2). Let $r, c_{4}$, and $C_{4}$ be defined as in Theorem 1 and assume that $h$ is sufficiently small. Then there exists a unique minimizer, $\mathbf{x}_{*}^{h}$, in $\overline{\mathcal{B}}^{h}\left(\mathbf{x}_{*}, r\right)$ of $\mathcal{F}(\mathbf{x} ; \mathbf{g})$. It is characterized by $\nabla^{h} \mathcal{F}\left(\mathbf{x}_{*}^{h} ; \mathbf{g}\right)=\mathbf{0}$, that is, it is the unique grid $h$ critical point in $\overline{\mathcal{B}}^{h}\left(\mathbf{x}_{*}, r\right)$.

Proof. For $\mathbf{x}=\mathbf{x}^{h} \in \overline{\mathcal{B}}^{h}\left(\mathbf{x}_{*}, r\right) \subset \overline{\mathcal{B}}\left(\mathbf{x}_{*}, r\right)$, Theorem 2 yields

$$
\frac{1}{2} c_{4}\left\|\mathbf{x}^{h}-\mathbf{x}_{*}\right\|_{1, \Omega}^{2} \leq \mathcal{F}\left(\mathbf{x}^{h} ; \mathrm{g}\right) \leq \frac{1}{2} C_{4}\left\|\mathbf{x}^{h}-\mathbf{x}_{*}\right\|_{1, \Omega}^{2} .
$$

We first prove that the minimizer over $\overline{\mathcal{B}}^{h}\left(\mathbf{x}_{*}, r\right)$, which exists by compactness, is actually in $\mathcal{B}^{h}\left(\mathbf{x}_{*}, r\right)$. To this end, it suffices to show that there exist an $\mathbf{x}^{h} \in \mathcal{B}^{h}\left(\mathbf{x}_{*}, r\right)$ that has a smaller functional value than the minimum of $\mathcal{F}(\mathbf{x} ; \mathbf{g})$ on $\partial \mathcal{B}^{h}\left(\mathbf{x}_{*}, r\right)$. To prove uniqueness of the minimizer, we then use an argument similar to that in Lemma 4.1 .

Any $\mathbf{x}_{\partial}^{h} \in \partial \mathcal{B}^{h}\left(\mathbf{x}_{*}, r\right)$ must satisfy $\left\|\mathbf{x}_{\partial}^{h}-\mathbf{x}_{*}\right\|_{1, \Omega}^{2}=r^{2}$. Hence, by (4.1), we have

$$
\mathcal{F}\left(\mathbf{x}_{\partial}^{h} ; \mathrm{g}\right) \geq \frac{1}{2} c_{4}\left\|\mathbf{x}_{\partial}^{h}-\mathbf{x}_{*}\right\|_{1, \Omega}^{2}=\frac{1}{2} c_{4} r^{2}
$$


for all $\mathbf{x}_{\partial}^{h} \in \partial \mathcal{B}^{h}\left(\mathbf{x}_{*}, r\right)$. Now let $r_{1}=\sqrt{c_{4} / C_{4}} r$ and assume that $h$ is so small that $\overline{\mathcal{B}}^{h}\left(\mathbf{x}_{*}, r_{1}\right)$ is not empty. Again by (4.1), any $\mathbf{x}^{h} \in \mathcal{B}^{h}\left(\mathbf{x}_{*}, r_{1}\right) \subset \mathcal{B}^{h}\left(\mathbf{x}_{*}, r\right)$ must satisfy

$$
\mathcal{F}\left(\mathbf{x}^{h} ; \mathrm{g}\right) \leq \frac{1}{2} C_{4}\left\|\mathbf{x}^{h}-\mathbf{x}_{*}\right\|_{1, \Omega}^{2}<\frac{1}{2} C_{4} r_{1}^{2}=\frac{1}{2} c_{4} r^{2} \leq \mathcal{F}\left(\mathbf{x}_{\partial}^{h} ; \mathrm{g}\right) .
$$

Therefore, the minimizer, $\mathbf{x}_{*}^{h}$, of $\mathcal{F}\left(\mathbf{x}^{h} ; \mathrm{g}\right)$ over $\overline{\mathcal{B}}^{h}\left(\mathbf{x}_{*}, r\right)$ must actually be in $\mathcal{B}^{h}\left(\mathbf{x}_{*}, r\right)$. Remark 2 confirms that it is a grid $h$ critical point: $\nabla^{h} \mathcal{F}\left(\mathbf{x}_{*}^{h} ; \mathbf{g}\right)=\mathbf{0}$.

To prove uniqueness, note that any other minimizer, $\mathbf{x}^{h}$, in $\mathcal{B}^{h}\left(\mathbf{x}_{*}, r\right)$ must be a grid $h$ critical point: $\nabla^{h} \mathcal{F}\left(\mathbf{x}^{h} ; \mathbf{g}\right)=\mathbf{0}$. It now suffices to show that $\mathbf{x}_{*}^{h}$ is the only grid $h$ critical point (which also proves the characterization assertion). To this end, note, for all $\mathbf{y}^{h} \in \mathcal{S}^{h}$, that

$$
\begin{aligned}
0 & =<\nabla^{h} \mathcal{F}\left(\mathbf{x}^{h} ; \mathbf{g}\right), \mathbf{y}^{h}>-<\nabla^{h} \mathcal{F}\left(\mathbf{x}_{*}^{h} ; \mathbf{g}\right), \mathbf{y}^{h}> \\
& =\mathcal{F}^{\prime}\left(\mathbf{x}^{h} ; \mathrm{g}\right)\left[\mathbf{y}^{h}\right]-\mathcal{F}^{\prime}\left(\mathbf{x}_{*}^{h} ; \mathbf{g}\right)\left[\mathbf{y}^{h}\right]=\mathcal{F}^{\prime \prime}\left(\tilde{\mathbf{x}}^{h} ; \mathbf{g}\right)\left[\mathbf{y}^{h}, \mathbf{x}^{h}-\mathbf{x}_{*}^{h}\right],
\end{aligned}
$$

for some $\tilde{\mathbf{x}}^{h} \in \mathcal{B}^{h}\left(\mathbf{x}_{*}, r\right)$. Again choosing $\mathbf{y}^{h}=\mathbf{x}^{h}-\mathbf{x}_{*}^{h} \in \mathcal{S}^{h} \subset \mathcal{V}$ and using Theorem 1 yields

$$
0=\mathcal{F}^{\prime \prime}\left(\tilde{\mathbf{x}}^{h} ; \mathbf{g}\right)\left[\mathbf{x}^{h}-\mathbf{x}_{*}^{h}, \mathbf{x}^{h}-\mathbf{x}_{*}^{h}\right] \geq c_{4}\left\|\mathbf{x}^{h}-\mathbf{x}_{*}^{h}\right\|_{1, \Omega}^{2},
$$

which establishes the result.

Lemma 4.3. Let $r, c_{4}$, and $C_{4}$ be defined as in Theorem 1 and choose any $r_{1}<\sqrt{c_{4} / C_{4}} r$. Then, for $\mathbf{x} \in \overline{\mathcal{B}}\left(\mathbf{x}_{*}, r_{1}\right)$, there exists a unique minimizer, $\mathbf{x}_{*}{ }^{2 h}=$ $\operatorname{argmin}_{\mathbf{x}^{2 h} \in \mathcal{S}^{2 h}, \mathbf{x}+\mathbf{x}^{2 h} \in \overline{\mathcal{B}}\left(\mathbf{x}_{*}, r\right)} \mathcal{F}\left(\mathbf{x}+\mathbf{x}^{2 h} ; \mathrm{g}\right)$. If $r$ is small enough, then this minimizer is characterized by $\nabla^{2 h} \mathcal{F}\left(\mathbf{x}+\mathbf{x}^{2 h} ; \mathrm{g}\right)=\mathbf{0}$, with $\mathbf{x}+\mathbf{x}^{2 h} \in \overline{\mathcal{B}}\left(\mathbf{x}_{*}, r\right)$ that is, it is the unique grid $2 h$ critical point for which $\mathbf{x}+\mathbf{x}^{2 h}$ stays in $\overline{\mathcal{B}}\left(\mathbf{x}_{*}, r\right)$. Thus, the result, $\mathbf{x}_{\frac{1}{2}}^{h}$, of STEP 1 of PML stays in $\overline{\mathcal{B}}^{h}\left(\mathbf{x}_{*}, r\right)$ for any initial guess, $\mathbf{x}_{0}^{h}$, in $\overline{\mathcal{B}}^{h}\left(\mathbf{x}_{*}, r_{1}\right)$.

Proof. The minimizer, $\mathbf{x}_{*}^{2 h}$, clearly exists by compactness. (Note that $\left\{\mathbf{x}+\mathcal{S}^{2 h}\right\} \cap$ $\overline{\mathcal{B}}\left(\mathbf{x}_{*}, r\right)$ is a nonempty set because it contains $\mathbf{x}=\mathbf{x}+0$.) To prove uniqueness and the fact that $\mathbf{x}_{*}^{2 h}$ is a grid $2 h$ critical point, first note that any $\mathbf{x}_{\partial} \in \partial \mathcal{B}\left(\mathbf{x}_{*}, r\right)$ must, by Theorem 2, satisfy $\mathcal{F}\left(\mathbf{x}_{\partial} ; \mathbf{g}\right) \geq \frac{1}{2} c_{4}\left\|\mathbf{x}_{\partial}-\mathbf{x}_{*}\right\|_{1, \Omega}^{2}=\frac{1}{2} c_{4} r^{2}$. Then, with $\mathbf{x} \in \overline{\mathcal{B}}\left(\mathbf{x}_{*}, r_{1}\right)$, again Theorem 2 implies that

$$
\mathcal{F}(\mathbf{x} ; \mathrm{g}) \leq \frac{1}{2} C_{4}\left\|\mathbf{x}-\mathbf{x}_{*}\right\|_{1, \Omega}^{2} \leq \frac{1}{2} C_{4} r_{1}^{2}<\mathcal{F}\left(\mathbf{x}_{\partial} ; \mathrm{g}\right) .
$$

Thus, $\mathcal{F}\left(\mathbf{x}+\mathbf{x}_{*}^{2 h} ; \mathrm{g}\right) \leq \mathcal{F}(\mathbf{x} ; \mathrm{g})<\mathcal{F}\left(\mathbf{x}_{\partial} ; \mathrm{g}\right)$, which implies that $\mathbf{x}+\mathbf{x}_{*}{ }^{2 h} \in \mathcal{B}\left(\mathbf{x}_{*}, r\right)$. Then $\mathbf{x}_{*}^{2 h}$ must satisfy the gradient condition, which follows by a similar standard argument similar to that of Remark 2.

Uniqueness and the characterization assertion can be now established as in the proofs of Lemmas 4.1 and 4.2. To this end, assume that there exists another minimizer, $\mathbf{x}+\mathbf{x}^{2 h} \in \mathcal{B}^{h}\left(\mathbf{x}_{*}, r\right)$, so that $\nabla^{2 h} \mathcal{F}\left(\mathbf{x}+\mathbf{x}^{2 h} ; \mathbf{g}\right)=\mathbf{0}$. It now suffices to show that this grid $2 h$ critical point condition implies that $\mathbf{x}^{2 h}=\mathbf{x}_{*}^{2 h}$ (which also proves the characterization assertion). To this end, note, for all $\mathbf{y}^{2 h} \in \mathcal{S}^{2 h}$, that

$$
\begin{aligned}
0 & =<\nabla^{2 h} \mathcal{F}\left(\mathbf{x}+\mathbf{x}^{2 h} ; \mathrm{g}\right), \mathbf{y}^{2 h}>-<\nabla^{2 h} \mathcal{F}\left(\mathbf{x}+\mathbf{x}_{*}{ }^{2 h} ; \mathbf{g}\right), \mathbf{y}^{2 h}> \\
& =\mathcal{F}^{\prime}\left(\mathbf{x}+\mathbf{x}^{2 h} ; \mathrm{g}\right)\left[\mathbf{y}^{2 h}\right]-\mathcal{F}^{\prime}\left(\mathbf{x}+\mathbf{x}_{*}{ }^{2 h} ; \mathbf{g}\right)\left[\mathbf{y}^{2 h}\right]=\mathcal{F}^{\prime \prime}\left(\mathbf{x}+\tilde{\mathbf{x}}^{2 h} ; \mathbf{g}\right)\left[\mathbf{y}^{2 h}, \mathbf{x}^{2 h}-\mathbf{x}_{*}{ }^{2 h}\right],
\end{aligned}
$$


for some $\mathbf{x}+\tilde{\mathbf{x}}^{2 h} \in \mathcal{B}^{h}\left(\mathbf{x}_{*}, r\right)$. As before, this leads to

$$
0=\mathcal{F}^{\prime \prime}\left(\mathbf{x}+\tilde{\mathbf{x}}^{2 h} ; \mathrm{g}\right)\left[\mathbf{x}^{2 h}-\mathbf{x}_{*}^{2 h}, \mathbf{x}^{2 h}-\mathbf{x}_{*}^{2 h}\right] \geq c_{4}\left\|\mathbf{x}^{2 h}-\mathbf{x}_{*}^{2 h}\right\|_{1, \Omega}^{2},
$$

which proves uniqueness and the characterization assertion.

The final claim follows simply by choosing $\mathbf{x}=\mathbf{x}^{h}$ and noting that we can choose $r>0$ so small that the nearest optimally corrected $\mathbf{x}^{h}$ must be the one in $\overline{\mathcal{B}}\left(\mathbf{x}_{*}, r\right)$.

TheOREM 4. Let $r$ and $r_{1}$ as in Lemma 4.3, define $r_{0}=\sqrt{c_{4} / C_{4}} r_{1}$, and choose $h$ and $\omega$ sufficiently small. Then, for any $\mathbf{x}_{0}^{h} \in \overline{\mathcal{B}}\left(\mathbf{x}_{*}, r_{0}\right)$, the PML iterates based on either (2.8) or (2.9) remain in $\overline{\mathcal{B}}^{h}\left(\mathbf{x}_{*}, r\right)$ and converge linearly with uniformly bounded factor according to

$$
\mathcal{F}\left(\mathrm{x}_{k+1}^{h} ; \mathrm{g}\right)-\mathcal{F}\left(\mathbf{x}_{*}^{h} ; \mathrm{g}\right) \leq \kappa\left(\mathcal{F}\left(\mathbf{x}_{k}^{h} ; \mathrm{g}\right)-\mathcal{F}\left(\mathbf{x}_{*}^{h} ; \mathrm{g}\right)\right), \quad k=0,1,2, \ldots,
$$

where $\kappa \in[0,1)$ depends only on $R e, r$, and $\Omega$.

Proof. We omit this fairly straightforward but somewhat lengthy proof and instead refer the reader to [10] for details.

\section{REFERENCES}

[1] P. Bochev, Z. Cai, T. Manteuffel, and S. McCormick, Analysis of velocity-flux first-order system least-squares principles for the Navier-Stokes equations: Part I, SIAM J. Numer. Anal, 35 (1998), pp. 990-1009.

[2] - Analysis of velocity-flux least-squares principles for the Navier-Stokes equations: Part II, SIAM J. Numer. Anal., 36 (1999), pp. 1125-1144.

[3] P. B. Bochev And M. D. Gunzburger, Finite element methods of least-squares type, SIAM Rev., 40 (1998), pp. 789-837 (electronic).

[4] S. C. Brenner And L. R. Scott, The Mathematical Theory of Finite Element Methods, vol. 15 of Texts in Applied Mathematics, Springer-Verlag, New York, 2002.

[5] V. Girault and P.-A. Raviart, Finite Element Methods for Navier-Stokes Equations, vol. 5 of Springer Series in Computational Mathematics, Springer-Verlag, Berlin, 1986. Theory and Algorithms.

[6] B.-N. JiAng, The least-squares finite element method, Scientific Computation, Springer-Verlag, Berlin, 1998. Theory and applications in computational fluid dynamics and electromagnetics.

[7] S.-D. Kim, C.-O. Lee, T. A. Manteuffel, S. F. McCormick, and O. Röhrle, First-order system least-squares functionals for the Oseen equations, Journal on Numerical Linear Algebra and Applications (JNLAA), submitted.

[8] T. A. Manteuffel, S. F. McCormick, O. Röhrle, and J. Ruge, Projection Multilevel Methods for Quasilinear Elliptic Partial Differential Equations: Numerical Results, SIAM J. Numer. Anal., accepted.

[9] S. F. MCConmick, Multilevel projection methods for partial differential equations, vol. 62 of CBMS-NSF Regional Conference Series in Applied Mathematics, Society for Industrial and Applied Mathematics (SIAM), Philadelphia, PA, 1992.

[10] O. RöHRLE, Multilevel First-Order System Least Squares for Quasilinear Elliptic Partial Differential Equations, PhD thesis, University of Colorado at Boulder, 2004.

[11] X.-C. TAI, Rate of convergence for some constraint decomposition methods for nonlinear variational inequalities, Numer. Math., 93 (2003), pp. 755-786.

[12] X.-C. TAI AND J. XU, Global and uniform convergence of subspace correction methods for some convex optimization problems, Math. Comp., 71 (2002), pp. 105-124 (electronic). 